\title{
Initial experience of a novel ergonomic surgical chair for laparoscopic pelvic surgery
}

\author{
Fernando J. Kim, David E. Sehrt, Wilson R. Molina, Jung-Sik Huh , Jens Rassweiler, Craig \\ Turner
}

Division of Urology, Department of Surgery, Denver Health Medical Center, Denver, CO, USA; Tony Grampsas Cancer Center, University of Colorado Health Science Center, Denver, CO, USA and SLK-Kliniken Heilbronn, Germany (JR)

\begin{abstract}
Introduction: We present the initial experience of a novel surgical chair for laparoscopic pelvic surgery, the ETHOS ${ }^{\mathrm{TM}}$ (Bridge City Surgical, Portland, OR).

Materials and Methods: The ETHOS chair has an adjustable saddle height that ranges from 0.89 to $1.22 \mathrm{~m}$ high, an overall width of $0.89 \mathrm{~m}$, and a depth of $0.97 \mathrm{~m}$. The open straddle is $0.53 \mathrm{~m}$ and fits most OR tables. We performed 7 pelvic laparoscopy cases with the $1^{\text {st }}$ generation ETHOS ${ }^{\mathrm{TM}}$ platform including 2 laparoscopic ureteral reimplantations, 5 laparoscopic pelvic lymphadenectomies for staging prostate cancer in which one case involved a laparoscopic radical retropubic prostatectomy, performed by 2 different surgeons.

Results: All 7 pelvic laparoscopic procedures were successful with the ETHOS ${ }^{\mathrm{TM}}$ chair. No conversion to open surgery was necessary. Survey done by surgeons after the procedures revealed minimal stress on back or upper extremities by the surgeons from these operations even when surgery was longer than 120 minutes. Conversely, the surgical assistants still had issues with their positions since they were on either sides of the patient stressing their positions during the procedure.

Conclusion: The ETHOS chair system allows the surgeon to operate seated in comfortable position with ergonomic chest, arms, and back supports. These supports minimize surgeon fatigue and discomfort during pelvic laparoscopic procedures even when these procedures are longer than 120 minutes without consequence to the patient safety or detrimental effects to the surgical team.
\end{abstract}

Key words: perineum; equipment and supplies; laparoscopy; surgical instruments; pelvis

Int Braz J Urol. 2011; 37: 455-460

\section{INTRODUCTION}

Present day surgeons are performing more minimally invasive procedures. The benefits of minimally invasive surgery (MIS) to patients include shortened hospital stay and recovery time as well as causing less scarring. Performing these procedures, however, often places physical demands on MIS surgeons that differ substantially from those of open surgery. These minimally in- vasive procedures have altered the way surgeons interact with the surgical field. Long shaft instruments, access ports, and the endoscope image display systems can cause numerous challenges for the surgeon's ergonomics in the OR (1). Unfortunately, the modern operating room has been slow to evolve to the new constraints these surgeons endure. The posture of surgeons has changed from a freedom of movement of open surgery to a straight and static posture during laparoscopy. The chronic static positioning of surgeons has 
been often related to discomfort. The upper extremities usually are in awkward and uncomfortable excursion for handling the long laparoscopic instruments, especially during pelvic procedures, where assistants and surgeons have to place themselves in a hip rotated position stressing the torso and the lower limbs. The upright posture during these procedures seems to be accompanied by the substantially less body movement and weight shifting then during open surgery (2). These observations suggest the advantage of supporting the surgeon's body during surgical procedures. The development of a novel surgical chair, the ETHOS (Bridge City Surgical, Portland, OR), was designed to improve the ergonomics of the laparoscopic pelvic procedures, effectively positioning the surgeon in the center of the patent's body.

\section{MATERIALS AND METHODS}

The ETHOS chair has an adjustable saddle height that ranges from 0.89 to $1.22 \mathrm{~m}$ high, an overall width of $0.89 \mathrm{~m}$, and a depth of 0.97 $\mathrm{m}$. The open straddle is $0.53 \mathrm{~m}$ and fits most OR tables. Two surgeons (FK and CT), and 2 surgical assistants performed 7 pelvic laparoscopic cases with the $1^{\text {st }}$ generation ETHOS ${ }^{\mathrm{TM}}$ platform including 2 laparoscopic ureteral reimplantations, 5 laparoscopic pelvic lymphadenectomies for staging prostate cancer, and a laparoscopic radical retropubic prostatectomy. Future procedures will be performed in 2011 with the new design of the new generation of ETHOS ${ }^{\mathrm{TM}}$ chair.

\section{RESULTS}

All demographic, operative and peri-operative data, and laboratory / pathological data are shown in Tables 1,2 and 3, respectively.

All 7 pelvic laparoscopic procedures were successful with the ETHOS ${ }^{\mathrm{TM}}$ chair. No conversion to open surgery was necessary. Survey done by surgeons after the procedures revealed minimal stress on back or upper extremities by the surgeons from these operations even when surgery was longer than 120 minutes. The surgeon dictated an effortlessness customization of the forearm, chest, and lower back supports which lead to nominal strain on his shoulders, back, and legs after each surgery. Table-4. Conversely, the surgical assistants still had issues with their positions since they were on either sides of the patient causing physical stress during the procedure. Discomfort was prominent during procedures that lasted more than 27 minutes. The areas affected were back, lower and upper limbs. Table-5. Integration of a Viky robotic camera driver (Endocontrol, Grenoble, France) was also used during the procedures. Specific measurements of the ETHOS ${ }^{\mathrm{TM}}$ chair are depicted in Figure-1. The Anesthesiologists survey with a short questionnaire on access to patient also reported no issues with the chair in terms of patient's safety at the end of each the procedure. Confidence was indicated by the surgeons that the surgical chair could easily be disengaged from the operating position if emergent access was required during the entirety of all procedures. The Anesthesiologists in these cases reported minimal interference from the chair's positioning. Easy access to the patient by the anesthesiologist achieved optimally deliver care. Table-6. Finally, the

Table 1 - Patient Demographic data.

\begin{tabular}{lccccc}
\hline Patient & Age(yr) & Sex & Height $\mathbf{( c m )}$ & Weight $\mathbf{( k g )}$ & BMI $\left(\mathbf{k g} / \mathbf{m}^{2}\right)$ \\
\hline 1 & 56 & Male & 185 & 71.1 & 21.26 \\
2 & 60 & Male & 152 & 63.0 & 19.92 \\
3 & 57 & Male & 182 & 83.3 & 24.89 \\
4 & Male & 170 & 75.6 & 26.1 \\
5 & 61 & Male & 175 & 87.3 & 28.42 \\
6 & 69 & Female & 155 & 67.0 & 27.93 \\
7 & 46 & Female & 160 & 69.3 & 27.3 \\
\hline
\end{tabular}


Table 2 - Peri-Operative Data.

\begin{tabular}{lccccc}
\hline Patient & Procedure & ASA & ORT $(\mathbf{m i n})$ & EBL $(\mathbf{m L})$ & Complications \\
\hline 1 & Lap. Lymphadenectomy and & 2 & 160 & 450 & None \\
& Lap. Prostatectomy & & & & \\
2 & Lap. Lymphadenectomy & 3 & 61 & Minimal & None \\
3 & Lap. Lymphadenectomy & 2 & 47 & Minimal & None \\
4 & Lap. Lymphadenectomy & 3 & 29 & Minimal & None \\
5 & Lap. Lymphadenectomy & 2 & 45 & 75 & None \\
6 & Ureteral Reimplantation & 3 & 77 & Minimal & None \\
\hline
\end{tabular}

Table 3 - Pathology Results in Patients that underwent Laparoscopic Pelvic/Obturator Lymphadenectomy.

\begin{tabular}{lcclc}
\hline Patient & PSA (ng/dL) & Gleason & Pathology & \# Lymphnodes \\
\hline 1 & 5.2 & $3+3$ & No cancer & 7 \\
2 & 5.4 & $3+4$ & No cancer & 5 \\
3 & 29.4 & $3+4$ & No cancer & 14 \\
4 & 7.2 & $3+4$ & No cancer & 7 \\
5 & 6.3 & $3+3$ & No cancer & 7 \\
\hline
\end{tabular}

nurses and OR staff were surveyed to express their concerns and satisfaction rates for space constraints, safety, appearance and mobility. Table-7.

\section{DISCUSSION}

Although while standing the human body appears static, continuous active control to maintain proper balance is always required. The body has been characterized as an unstable balance system, has twothirds of its mass located at two-thirds of its height above the ground (3). Posture during standing thus forms an inverted pendulum, appearing to be static but actually under dynamic control (4). Neck and shoulder pain have been exhibited by laparoscopic surgeons' and their posture $(5,6)$. In an assessment of fatigue of laparoscopic surgeons, the static contraction of the upper trapezius, middle trapezius, lower erector spinai, and hamstrings exceeded maximal voluntary isometric contraction threshold limits for a fatigue response. The median MVS fatigue threshold was also exceeded in the middle trapezius and the lower erector spinai (7). Correct posture is very important not only with minimizing physical discomfort but also with improving task performance (8).

In addition to poor posture, the surgeons also deal with problems related to non-optimal working

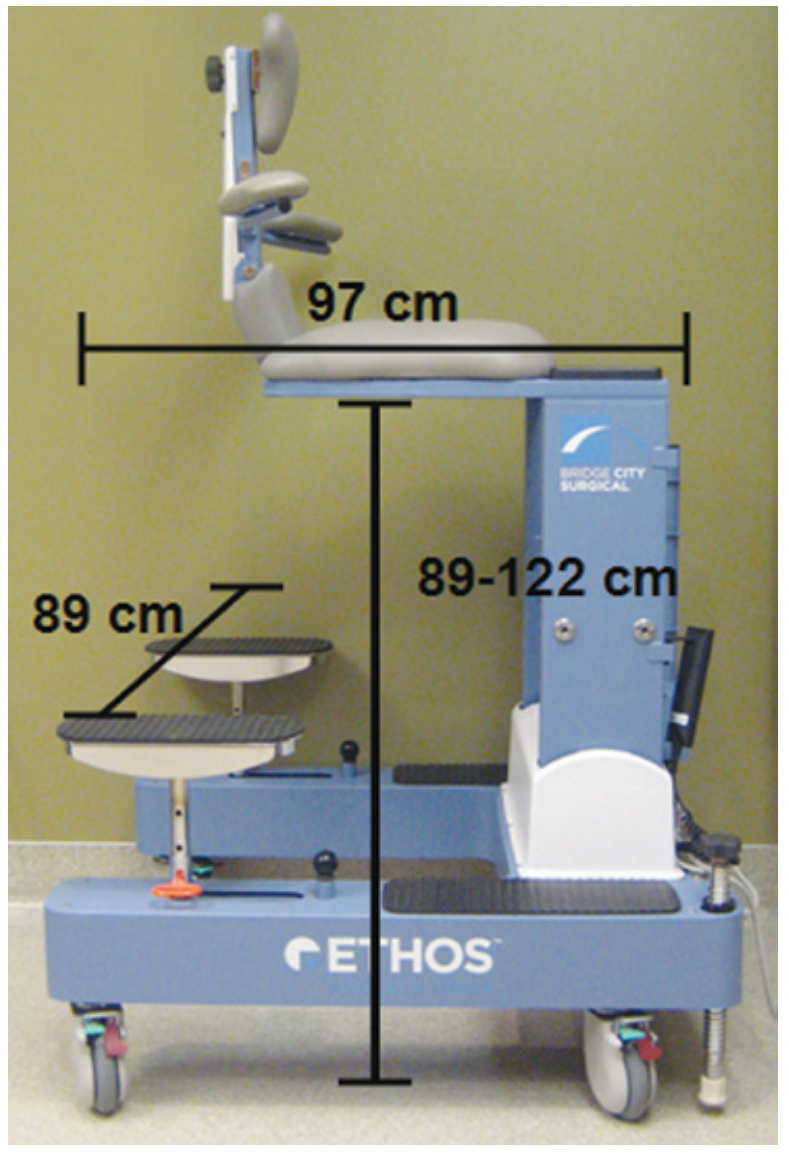

Figure 1 - Dimensions of the ETHOS Surgical Platform. 
Table 4 - Surgeon Post-Operative Muscular Discomfort Questionnaire.

\begin{tabular}{lccc}
\hline & Never & Sometimes & All the Time \\
\hline Abdominal & 2 & 0 & 0 \\
Back & 2 & 0 & 0 \\
Upper Limbs & 2 & 0 & 0 \\
Lower Limbs & 2 & 0 & 0 \\
\hline
\end{tabular}

Table 5 - Surgical Assistant on Post-Operative Muscular Discomfort Questionnaire.

\begin{tabular}{lccc}
\hline & Never & Sometimes & All the Time \\
\hline Abdominal & 4 & 0 & 0 \\
Back & 0 & 0 & 4 \\
Upper Limbs & 0 & 0 & 4 \\
Lower Limbs & 0 & 0 & 4 \\
\hline
\end{tabular}

Discomfort was prominent during procedures that lasted more than 27 minutes.

height. Unfavorable table height can increase the upper extremity effort and has potential for musculoskeletal injury. Operative tables were originally designed for open surgery and thus not optimal for MIS. Operating tables may not be capable of translating to the proper height at or $5-10 \mathrm{~cm}$ below the elbow of the surgeon $(9,10)$. In the study by Engsberg it was also shown that the anterior deltoid exceeds the static, median, and peak limits of maximal voluntary isometric contraction thresholds (7). Physical constraints on the shoulders and arms also contribute to the surgeon's fatigue.
The crowding in the operating room and the positioning of the surgical team around the operating table also contributes to poor ergonomics. Laparoscopic procedures have shown to significantly increase crowding in the room (11). The percentage of operating space occupied by equipment and personnel increased from $36 \%$ for open surgery to $41 \%$ for laparoscopy. The total count of equipment present in the operating room increased from 6 for open procedures to 13 for laparoscopic (11). The freedom of the positioning the surgical team and equipment around the operating table is limited because the operating table is fixated to the floor.

Table 6 - Anesthesiogist Access to Patient Questionnaire.

\begin{tabular}{lccc}
\hline & easy & Difficult & impossible \\
\hline Access to Face & 7 & \\
Access to Tubes & 7 & $2 *$ \\
Access to Wire & 5 & \\
Safe device to place above patient & 7 &
\end{tabular}

*due to surgical drapes covering the face. 
Table 7 - Anesthesiogist Access to Patient Questionnaire.

\begin{tabular}{lccc}
\hline & No & Yes & Do not know \\
\hline Space constraints & 5 & 0 & 0 \\
Safe & 5 & 0 & 0 \\
Pleasing appearance & 2 & 3 & 0 \\
Easy mobility & 2 & 3 & 0 \\
\hline
\end{tabular}

The ETHOS ${ }^{\mathrm{TM}}$ positions the surgeon in a chair above the patient with minimal interference to the surgical team. Crowding is therefore minimized by moving the surgeon from tableside to above the patient. Forearms, chest, and lower back support with adjustable locking mechanisms are customizable to the surgeon's size and preferred positioning. Manipulation of laparoscopic instruments can occur without stressing the surgeon's back, upper, or lower limbs. Moreover, the disengagement mechanism of the chair from the operative position would allow emergent access if needed maintaining patient safety.

\section{CONCLUSIONS}

The ETHOS ${ }^{\mathrm{TM}}$ system enables the surgeon to operate seated in comfortable position with ergonomic chest, arms, and back support effectively reducing muscle activity and discomfort which portends to improve surgeon satisfaction. Although many Urologists have adapted use of the da Vinci robotic surgical system to overcome the aforementioned ergonomic challenges; the cost of the robotic system, steep learning curve, and remote surgeon location are substantial issues that must be considered. The ETHOSTM system avoids these issues. Cost is below these robotic systems, surgeons use laparoscopic tools they have familiarity with, and the ETHOS places the surgeon in the orthotopic position above the patient. Although further clinical validation is needed; the ergonomics of the ETHOS chair for minimally invasive procedures will improve conditions for laparoscopic surgeons by overcoming the constraints of robotic and conventional bedside surgery.

\section{CONFLICT OF INTEREST}

None declared.

\section{REFERENCES}

1. van Det MJ, Meijerink WJ, Hoff C, Totté ER, Pierie JP. Optimal ergonomics for laparoscopic surgery in minimally invasive surgery suites: a review and guidelines. Surg Endosc. 2009; 23: 1279-85.

2. Berguer R, Rab GT, Abu-Ghaida H, Alarcon A, Chung J. A comparison of surgeons' posture during laparoscopic and open surgical procedures. Surg Endosc. 1997; 11: 139-42.

3. Winter DA. Human balance and posture control during standing and walking. Gait Posture. 1995; 3: 193-214.

4. Gage WH, Winter DA, Frank JS, Adkin AL. Kinematic and kinetic validity of the inverted pendulum model in quiet standing. Gait Posture. 2004; 19: 124-32.

5. Berguer R, Forkey DL, Smith WD. Ergonomic problems associated with laparoscopic surgery. Surg Endosc. 1999; 13: 466-8.

6. Wauben LS, van Veelen MA, Gossot D, Goossens RH. Application of ergonomic guidelines during minimally invasive surgery: a questionnaire survey of 284 surgeons. Surg Endosc. 2006; 20: 1268-74.

7. Uhrich ML, Underwood RA, Standeven JW, Soper NJ, Engsberg JR. Assessment of fatigue, monitor placement, and surgical experience during simulated laparoscopic surgery. Surg Endosc. 2002; 16: 635-9.

8. Bhatnager V, Drury CG, Schiro SG. Posture, postural discomfort, and performance. Hum Factors. 1985; 27: 189-99.

9. Manasnayakorn S, Cuschieri A, Hanna GB. Ergonomic assessment of optimum operating table height for hand-assisted laparoscopic surgery. Surg Endosc. 2009; 23: 783-9. 
10. Berquer R, Smith WD, Davis S. An ergonomic study of the optimum operating table height for laparoscopic surgery. Surg Endosc. 2002; 16: 416-21.
11. Alarcon A, Berguer R. A comparison of operating room crowding between open and laparoscopic operations. Surg Endosc. 1996; 10: 916-9.

\section{Correspondence address:}

Dr. Fernando J. Kim

Chief of Urology, DHMC

Associate Professor of Surgery/Urology, UCHSC

Director of Minimally Invasive Urological Oncology

Accepted after revision:

May 05, 2011

Tony Grampsas Cancer Center, UCHSC

E-mail: fernando.kim@dhha.org 